\title{
Planar Point Sets With Large Minimum Convex Decompositions
}

\author{
Jesús García-López • Carlos M. Nicolás
}

\begin{abstract}
We show the existence of sets with $n$ points $(n \geq 4)$ for which every convex decomposition contains more than $\frac{35}{32} n-\frac{3}{2}$ polygons, which refutes the conjecture that for every set of $n$ points there is a convex decomposition with at most $n+C$ polygons. For sets having exactly three extreme points we show that more than $n+\sqrt{2(n-3)}-4$ polygons may be necessary to form a convex decomposition.
\end{abstract}

Keywords Convex decompositions $\cdot$ Triangulations $\cdot$ Empty polygons

\section{Introduction}

Let $V$ be a finite set of points in general position in the plane, i.e., no three points of $V$ lie on a straight line. A convex subdivision of $V$ is a set of convex polygons $\left\{P_{1}, \ldots, P_{k}\right\}$ with vertices in $V$ such that $\cup_{i} P_{i}=\operatorname{conv}(V)$ and $P_{i} \cap P_{j}$ is a (possibly empty) face of both $P_{i}$ and $P_{j}$ (see [6]). In this paper we impose the following additional condition: the polygons $P_{i}$ must be empty, i.e., no element of $V$ is contained in the interior of any of the polygons. This condition is equivalent (for points in general position) to the 
requirement that every element in $V$ should be a vertex of at least one polygon in the subdivision. We use the name convex decomposition (or just decomposition) to refer to this special type of subdivision.

The number of polygons in a decomposition is called the size of the decomposition. Let $G(V)$ be the minimum size among the decompositions of $V$. Let $g(n)$ be the maximum value of $G(V)$ among the sets $V$ of $n$ points in general position in the plane. Aichholzer and Krasser [2] showed $g(n) \geq n+2$. On the other hand we have the trivial bound $g(n) \leq 2 n-5$ for $n \geq 3$ (by considering triangulations). Hosono [5] proved $g(n) \leq\lceil(7 / 5)(n-3)\rceil+1$. Sakai and Urrutia [7] announced the bound $g(n) \leq(4 / 3) n-2$. Rivera-Campo and Urrutia conjectured that $g(n) \leq n+C$ for some constant $C$ (see Conjecture 6 in Section 8.5 of [3]). It is clear that if a set $V$ with $n$ vertices admits a decomposition into convex quadrilaterals then $G(V) \leq n$. Thus, roughly speaking a formula of the type $g(n) \leq n+C$ for constant $C$ would indicate that the triangles that we may be forced to use to obtain a convex decomposition of $V$ can be offset by sufficiently many polygons with five or more faces. In this paper we refute this conjecture by showing that $g(n)>(35 / 32) n-3 / 2$ for $n \geq 4$. We present an improved, simpler version (with corrected proofs) of the construction in our unpublished draft [4] (cited in [1,5]).

We refer to [1] for bounds on related objects such as pseudo-convex decompositions and convex and pseudo-convex partitions and coverings.

\section{Basic Construction}

First we review the idea of contraction in the context of decompositions. It is convenient when performing contractions to identify a decomposition $S$ with the geometric graph consisting of the vertices and sides of the polygons in $S$. Let $V$ be a finite subset of $\mathbb{R}^{2}$. Let $p \in \mathbb{R}^{2}-V$ and suppose that $V \cup\{p\}$ is in general position. Define cell $(p, V)$ as the cell that contains $p$ in the line arrangement determined by $V$, i.e., $x \in \operatorname{cell}(p, V)$ if and only if $x$ and $p$ are on the same side of $l$ for every line $l$ through any two points in $V$. In other words, $V \cup\{x\}$ and $V \cup\{p\}$ have the same order type. It is clear that if $S$ is a convex decomposition of $V \cup\{x\}$ and $x \in \operatorname{cell}(p, V)$ then there exists a decomposition $S^{\prime}$ of $V \cup\{p\}$ which is combinatorially equivalent to $S$. More in general, if $S$ is a decomposition of $V \cup V_{1}$ and $V_{1} \subset \operatorname{cell}(p, V)$, where $p$ may be contained in $V_{1}$ but not in $V$, we obtain from $S$ a decomposition of $V \cup\{p\}$ if we contract every element of $V_{1}$ to $p$.

We work in the more general setting of $\mathbb{R}^{2} \cup\left\{p_{\infty}\right\}$, where $p_{\infty}$ is the point at infinity in the direction of the positive $x$-axis. An edge between a vertex $v$ of $\mathbb{R}^{2}$ and $p_{\infty}$ is simply an infinite ray that starts at $v$ and extends in the positive horizontal direction. If $V \subset \mathbb{R}^{2}$ then $V \cup\left\{p_{\infty}\right\}$ is in general position if $V$ is in general position and no two vertices of $V$ have the same $y$ coordinate. Suppose $V \subset \mathbb{R}^{2}$ and let $V \cup\left\{p_{\infty}\right\}$ be in general position. Define cell $\left(p_{\infty}, V\right)$ as the unbounded region determined by the following rule: $x \in \operatorname{cell}\left(p_{\infty}, V\right)$ if and only if for every line $l$ through two points in $V, x$ lies above $l$ if $l$ has negative slope and below $l$ if $l$ has positive slope. If $V_{1} \subset \operatorname{cell}\left(p_{\infty}, V\right)$ and $S$ is a decomposition of $V \cup V_{1} \cup\left\{p_{\infty}\right\}$ (note $p_{\infty}$ is not contained in $V_{1}$ nor $V$ ) we obtain a decomposition of $V \cup\left\{p_{\infty}\right\}$ by contracting $V_{1}$ to 
(a)

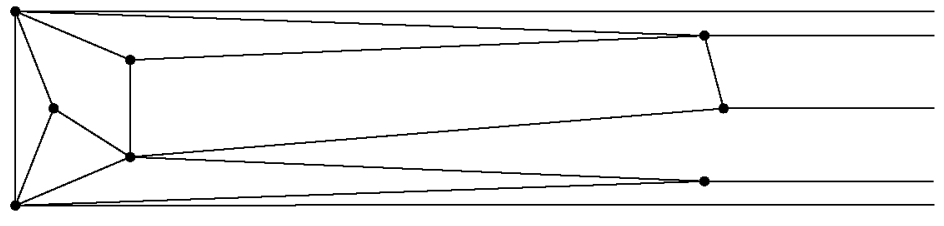

(b)

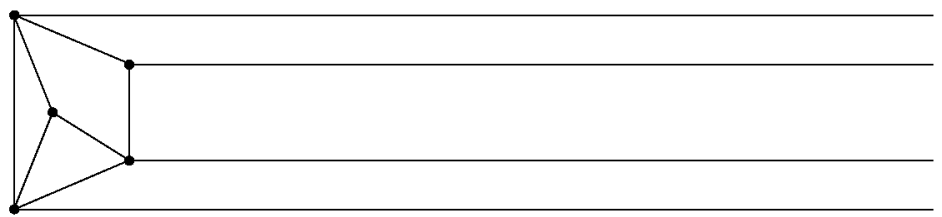

Fig. 1 a A decomposition of $V \cup V_{1} \cup\left\{p_{\infty}\right\}$, where $V_{1}$ is the set containing the three points shown on the right. b A decomposition of $V \cup\left\{p_{\infty}\right\}$, obtained by contracting $V_{1}$ to $p_{\infty}$ in a

$p_{\infty}$. In this case the contraction amounts to deleting $V_{1}$ and every edge incident to it, and adding an edge (i.e., ray) between $v$ and $p_{\infty}$ for each $v \in V$ adjacent to a vertex in $V_{1}$. See Fig. 1 .

Let $B=\{(0,0),(0,1)\}$ and $B^{*}=B \cup\left\{p_{\infty}\right\}$. We consider first decompositions of the convex hull of $B^{*}$ (the "triangle" bounded by the lines $y=0, x=0$ and $y=1$ ).

Inductively, define the sets $L_{0}, \ldots, L_{k+1}$ as follows. Let $L_{0}=\emptyset, L_{1}=\left\{\left(1, \frac{1}{2}\right)\right\}$ and suppose the sets $L_{0}, \ldots, L_{k}, k \geq 1$, have been chosen so that the following properties hold for $0 \leq i<k$ :

(i) $L_{i+1} \subset \operatorname{cell}\left(p_{\infty}, B \cup L_{1} \cup \ldots \cup L_{i}\right)$.

(ii) The vertices in $L_{i+1}$ form a vertical layer concave to the left, i.e., every vertex $v$ in $L_{i+1}$ is contained in a line having $B \cup L_{1} \cup \cdots \cup L_{i+1}-\{v\}$ on one side and $p_{\infty}$ on the other side. This is equivalent (by property (i)) to requiring the set $B \cup L_{i+1}$ to be in convex position.

(iii) $\left|L_{i+1}\right|=i+1$. Additionally, when ordered according to their $y$-coordinates the elements of $L_{i}$ and $L_{i+1}$ alternate.

(iv) $B^{*} \cup L_{1} \cup \ldots \cup L_{i+1}$ is in general position.

Then $L_{k+1}$ can be chosen so that properties (i)-(iv) are satisfied for $0 \leq i<k+1$ : on account of the general position $\operatorname{cell}\left(p_{\infty}, B \cup L_{1} \cup \ldots \cup L_{k}\right)$ contains an infinite rectangle of height 1 , i.e., there exists a number $M$ such that the convex hull of $\left\{(M, 0),(M, 1), p_{\infty}\right\}$ is contained in $\operatorname{cell}\left(p_{\infty}, B \cup L_{1} \cup \ldots \cup L_{k}\right)$. For $i \in\{1, \ldots, k+1\}$ choose $y_{i}$ in the open interval $\left(\bar{y}_{i-1}, \bar{y}_{i}\right)$ where $\bar{y}_{1}, \ldots, \bar{y}_{k}$ are the $y$-coordinates in increasing order of the $k$ points in $L_{k}$ and $\bar{y}_{0}=0, \bar{y}_{k+1}=1$. Also choose the numbers $y_{i}$ to be distinct from all the $y$-coordinates of the points in $L_{1} \cup \cdots \cup L_{k}$ to ensure general position. Now choose $x_{i}>M$ such that the points $\left(x_{i}, y_{i}\right)$ form a vertical layer concave to the left (as defined in (ii)) while preserving the general position, and let $L_{k+1}=\left\{\left(x_{1}, y_{1}\right), \ldots,\left(x_{k+1}, y_{k+1}\right)\right\}$.

For every $k \geq 1$, denote the set $L_{1} \cup \cdots \cup L_{k}$ by $C_{k}$. See Fig. 2 . 


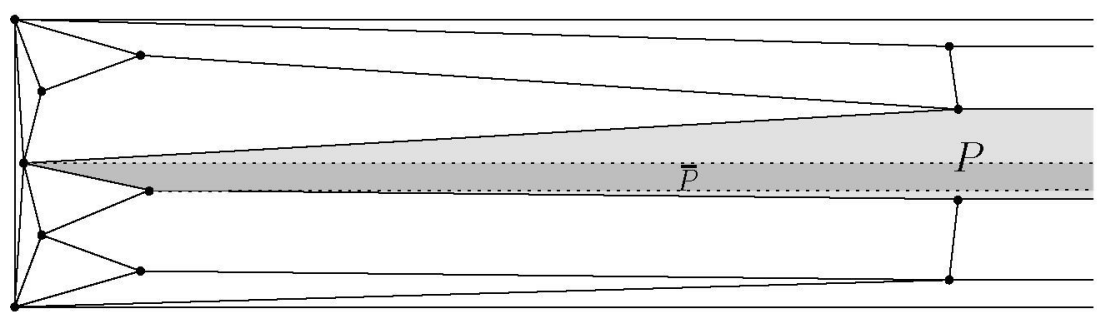

Fig. 2 A decomposition of $B^{*} \cup C_{4}$. $\bar{P}$ is obtained from $P$ when $L_{4}$ is contracted to $p_{\infty}$. Note that $P$ is incident to $p_{\infty}$, hence $\bar{P} \subseteq P$

\section{Results}

We are interested in the sets $B^{*} \cup C_{k}$ because the discrepancy between its size and the size of its decompositions is large. Define $\Delta(S)$ for any decomposition $S$ of a set of vertices $V$ to be $|S|-\left|V_{\text {int }}\right|$, i.e., the difference between the number of polygons in the decomposition and the number of vertices of $V$ that lie in the interior of $\operatorname{conv}(V)$. Let $R_{\infty}(S)$ be the set of polygons in $S$ incident to $p_{\infty}$.

Theorem 1 If $S$ is a decomposition of $B^{*} \cup C_{k}$ then $\Delta(S) \geq\left|R_{\infty}(S)\right|$.

Proof 1 By induction on $k$. The case $k=1$ is clear since the only decomposition $S$ of $B^{*} \cup L_{1}$ satisfies $|S|=3,\left|R_{\infty}(S)\right|=2$ and there is one interior vertex.

Assume the theorem is true for $k \geq 1$. Let $S$ be a decomposition of $B^{*} \cup C_{k+1}$. Since $L_{k+1} \subset \operatorname{cell}\left(p_{\infty}, B \cup C_{k}\right)$ we obtain a decomposition $\bar{S}$ of $B^{*} \cup C_{k}$ by contracting $L_{k+1}$ to $p_{\infty}$. For $P \in S$ let $\bar{P}$ be the polygon obtained from $P$ after contracting $L_{k+1}$ to $p_{\infty}$. Note that $\bar{P}$ may be a degenerate polygon (i.e., with empty interior) consisting only of the point $p_{\infty}$ or of a horizontal ray joining a point to $p_{\infty}$. Clearly $\bar{P}$ is degenerate if and only if $P$ is incident to at most one point in $B \cup C_{k}$. Let $R^{\text {new }}(S)$ be the subset of $S$ consisting of the polygons $P$ for which $\bar{P}$ is degenerate. The map $P \rightarrow \bar{P}$ restricted to $S-R^{\text {new }}$ establishes a bijection with $\bar{S}$, so $|S|=|\bar{S}|+\left|R^{\text {new }}(S)\right|$. If we think of the decomposition $S$ as being obtained from $\bar{S}$ by reversing the contraction then the polygons in $R^{\text {new }}$ are the new polygons that have not been counted in $\bar{S}$. Applying the inductive hypothesis we obtain

$$
\begin{aligned}
\Delta(S) & =|S|-\left|C_{k+1}\right|=|\bar{S}|+\left|R^{\text {new }}(S)\right|-\left|C_{k}\right|-\left|L_{k+1}\right| \\
& =\Delta(\bar{S})+\left|R^{\text {new }}(S)\right|-k-1 \geq\left|R_{\infty}(\bar{S})\right|+\left|R^{\text {new }}(S)\right|-k-1
\end{aligned}
$$

Note that if $P \in R_{\infty}(S)-R^{\text {new }}(S)$ then $\bar{P} \in R_{\infty}(\bar{S})$ since an unbounded polygon (i.e., incident to $p_{\infty}$ ) either becomes degenerate or remains unbounded after the contraction. But we also have elements in $R_{\infty}(\bar{S})$ that come from bounded polygons in $S$. Let $R^{\text {bnd }}(S)$ be the set of polygons $P$ in $S-R_{\infty}(S)$ such that $\bar{P} \in R_{\infty}(\bar{S})$. Then $\left|R_{\infty}(\bar{S})\right|=\left|R^{\text {bnd }}(S)\right|+\left|R_{\infty}(S)-R^{\text {new }}(S)\right|$. Substituting in (1) we obtain

$$
\begin{aligned}
\Delta(S) & \geq\left|R^{\text {bnd }}(S)\right|+\left|R_{\infty}(S)-R^{\text {new }}(S)\right|+\left|R^{\text {new }}(S)\right|-k-1 \\
& \geq\left|R^{\text {bnd }}(S)\right|+\left|R_{\infty}(S)\right|-k-1
\end{aligned}
$$


Therefore the result follows if we show that $\left|R^{\text {bnd }}(S)\right| \geq k+1$. Now, for each vertex $v$ in $L_{k+1}$ there is a polygon $Q_{v}$ in $R_{\infty}(\bar{S})$ that contains $v$ in its interior (since the polygons in $\bar{S}$ cover conv $\left(B^{*}\right)$ and $L_{k+1} \subset \operatorname{cell}\left(p_{\infty}, B \cup C_{k}\right)$ ). Let $P_{v}$ be the polygon in $S$ such that $\bar{P}_{v}=Q_{v}$. We want to show that $P_{v} \notin R_{\infty}(S)$ and that $P_{v} \neq P_{v^{\prime}}$ for $v \neq v^{\prime}$. Suppose $P_{v} \in R_{\infty}(S)$. The two rays incident to $p_{\infty}$ on the boundary of $P_{v}$ are horizontal, therefore, by convexity, every vertex of $P_{v}$ lies either between the two parallel lines defined by these rays or at the endpoint of one of the rays. Hence, $P_{v} \in R_{\infty}(S)$ implies $\bar{P}_{v} \subseteq P_{v}$ (see Fig. 2). Since $v$ belongs to the interior of $\bar{P}_{v}$ and $\bar{P}_{v} \subseteq P_{v}$ it follows that $v$ belongs to the interior of $P_{v}$, which contradicts the fact that $P_{v}$ is an empty polygon. Therefore $P_{v} \in R^{\text {bnd }}(S)$ for all $v \in L_{k+1}$.

Finally suppose $P_{v}=P_{v^{\prime}}$ for some $v, v^{\prime} \in L_{k+1}, v \neq v^{\prime}$. Then $\bar{P}_{v}=\bar{P}_{v^{\prime}}$ so $\bar{P}_{v}$ contains both $v$ and $v^{\prime}$ in its interior. By its convexity $\bar{P}_{v}$ contains the segment joining $v$ and $v^{\prime}$. But there is a vertex $w$ in $L_{k}$ whose $y$ coordinate lies between the $y$ coordinates of $v$ and $v^{\prime}$ (the coordinates of $L_{k}$ and $L_{k+1}$ alternate). Since $L_{k}$ is concave to the left $w$ has to be incident to $p_{\infty}$ in the decomposition $\bar{S}$. Thus, the ray coming out of $w$ intersects the segment between $v$ and $v^{\prime}$ which is supposed to be contained in the interior of $\bar{P}_{v}$. This contradiction shows that $P_{v} \neq P_{v^{\prime}}$ for $v \neq v^{\prime}$. Hence $\left|R^{\text {bnd }}(S)\right| \geq k+1$ and the result follows from (2).

Corollary 2 For any decomposition $S$ of $B^{*} \cup C_{k}, \Delta(S) \geq k+1$.

Proof $2 L_{k}$ is concave to the left. Therefore every $v \in L_{k}$ is incident to $p_{\infty}$, so $R_{\infty}(S) \geq k+1$.

Corollary 3 For any decomposition $S$ of $B^{*} \cup C_{k}$, the number of polygons not incident to $p_{\infty}$ is at least $\left|C_{k}\right|$.

Recall that $G(V)$ is defined as the minimum size of a decomposition of $V$ and $g(n)$ as the maximum value of $G(V)$ among all the sets with $n$ elements in general position. Let $g_{3}(n)$ be the maximum value of $G(V)$ among the sets $V \subset \mathbb{R}^{2}$ in general position having $n$ elements of which exactly three lie on the boundary of $\operatorname{conv}(V)$. In order to obtain a bound for $g_{3}$ we replace $p_{\infty}$ by an actual point on $\mathbb{R}^{2}$. Let $z_{k} \in \operatorname{cell}\left(p_{\infty}, B \cup C_{k}\right)$. It is clear that any decomposition of $B \cup C_{k} \cup\left\{z_{k}\right\}$ yields a decomposition of $B^{*} \cup C_{k}$ with the same number of polygons when $z_{k}$ is contracted to $p_{\infty}$. Combined with Corollary 2 we obtain the following bound on $g_{3}$ :

Theorem $4 g_{3}\left(3+\left|C_{k}\right|\right) \geq\left|C_{k}\right|+k+1$.

In order to obtain a result valid for every $n$, observe that $g_{3}(n+1) \geq g_{3}(n)+1$ (i.e., $g_{3}$ is strictly monotonic). Indeed, let $V$ be a set of $n$ points of which exactly three, $b_{1}, b_{2}$ and $b_{3}$, lie on the boundary of $\operatorname{conv}(V)$ and such that $G(V)=g_{3}(n)$. Let $V^{\prime}$ be the set $V \cup\{w\}$ where $w$ satisfies $w \in \operatorname{conv}(V) \cap \operatorname{cell}\left(b_{1}, V-\left\{b_{1}\right\}\right)$ and the line through $b_{1}$ and $w$ separates $b_{2}$ from the rest of the points in $V\left(w\right.$ is very close to the side $b_{1} b_{2}$ and to the vertex $b_{1}$ ). Then every decomposition of $V^{\prime}$ contains the triangle with vertices $b_{1}, b_{2}$ and $w$. Moreover, every decomposition $S$ of $V^{\prime}$ produces a decomposition $\bar{S}$ of $V$ when $w$ is contracted to $b_{1}$. Since the triangle $b_{1} b_{2} w$ collapses after the contraction we obtain $|S|>|\bar{S}| \geq|G(V)|$. Therefore, $g_{3}(n+1) \geq G\left(V^{\prime}\right)>G(V)=g_{3}(n)$. Taking $V$ to be a set such that $g(n)=G(V)$ and proceeding in a similar way we also obtain the monotonicity of the function $g(n)$. 
Fig. 3 A decomposition of the set $D_{4,2}$

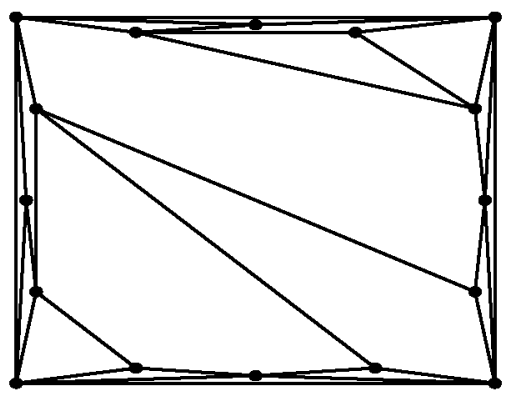

Theorem $5 g_{3}(n+j) \geq g_{3}(n)+j$ and $g(n+j) \geq g(n)+j$.

Let $n_{k}=3+\left|C_{k}\right|=3+k(k+1) / 2$. Solving for $k$ we obtain $k=(1 / 2)(-1+$ $\left.\sqrt{1+8\left(n_{k}-3\right)}\right)$. Therefore, taking $n_{k} \leq n<n_{k+1}$ we have $k \leq(1 / 2)(-1+$ $\sqrt{1+8(n-3)})<k+1$. From this inequality and Theorems 4 and 5 we obtain the following bound on $g_{3}$ :

Theorem 6 For all $n \geq 3, g_{3}(n)>n-4+\sqrt{2(n-3)}$.

Proof 3 Let $n_{k} \leq n<n_{k+1}$. By Theorems 4 and 5, $g_{3}(n) \geq g_{3}\left(n_{k}\right)+n-n_{k} \geq$ $n_{k}-3+k+1+n-n_{k}$. But $k+1>(1 / 2)(-1+\sqrt{1+8(n-3)})$, so $g_{3}(n)>$ $n-3+(1 / 2)(-1+\sqrt{1+8(n-3)})>n-4+\sqrt{2(n-3)}$.

Now we consider a more general construction that yields a bound for the function $g(n)$. Let $P=\left\{v_{0}, \ldots, v_{h-1}\right\}$ be the vertices of a regular $h$-gon listed in clockwise order. Let $B_{i}$ denote the set $\left\{v_{i-1}, v_{i}\right\}$ (all indices from now on are computed modulo $h$ ). Let $\bar{B}_{i}$ be the segment with endpoints $v_{i-1}$ and $v_{i}$. Let $p_{\infty}^{i}$ be the point at infinity in the direction perpendicular to $\bar{B}_{i}$ on the side containing conv $(P)$. We work with $p_{\infty}^{i}$ in the same way we did with $p_{\infty}$. For example, cell $\left(p_{\infty}^{i}, V\right)$ (for $V \subset \mathbb{R}^{2}$ ) is the unbounded region that corresponds to $\operatorname{cell}\left(p_{\infty}, \bar{V}\right)$ when the plane is rotated so that $p_{\infty}^{i}$ corresponds to $p_{\infty}$ and $V$ to $\bar{V}$.

Now apply appropriate affine transformations to $C_{k}$ in order to obtain, for each $i$, sets $C_{k}^{i}$ satisfying the following property:

$$
\bigcup_{j \neq i} C_{k}^{j} \cup P-B_{i} \subset \operatorname{cell}\left(p_{\infty}^{i}, B_{i} \cup C_{k}^{i}\right)
$$

In other words, rotate and scale $C_{k}$ until the set $B_{i}$ plays the role of $B$ in our previous construction and then affinely compress $C_{k}$ as much as necessary toward the segment $\bar{B}_{i}$ to obtain a set $C_{k}^{i}$ such that cell $\left(p_{\infty}^{i}, B_{i} \cup C_{k}^{i}\right)$ contains all of conv $(P)$ except for $\bar{B}_{i}$ and a small neighborhood around it. Let $D_{h, k}=P \cup \cup_{i} C_{k}^{i}$. See Fig. 3 for an example.

Let $S$ be a decomposition of $D_{h, k}$. From (3) it follows that if we contract every vertex not in $B_{i} \cup C_{k}^{i}$ to $p_{\infty}^{i}$ we obtain a decomposition which is combinatorially equivalent to a decomposition of $B^{*} \cup C_{k}$. In particular, from Corollary 3 we see that in any decomposition $S$ of $D_{h, k}$ and for each $i$ the number of polygons incident only to vertices in $B_{i} \cup C_{k}^{i}$ is at least $k(k+1) / 2$. In addition to these $h k(k+1) / 2$ polygons, 
$S$ must contain at least $h k / 2$ more polygons which are incident to the diagonals that connect the last layer of each $C_{k}^{i}$ with vertices outside $B_{i} \cup C_{k}^{i}$ (there are at least $h k / 2$ such diagonals). Therefore, for every decomposition $S$ of $D_{h, k},|S| \geq h k(k+2) / 2$. Hence,

Theorem $7 G\left(D_{h, k}\right) \geq h k(k+2) / 2$, for $h \geq 3, k \geq 1$.

The quotient between $h k(k+2) / 2$ and $\left|D_{h, k}\right|$ does not depend on $h$ and attains a maximum, among integer values of $k$, at $k=5$. Since $\left|D_{h, 5}\right|=16 h$ the previous theorem yields $g(n) \geq(35 / 32) n$ when $n=16 h, h \geq 3$. For arbitrary $n$, we obtain the following theorem:

Theorem $8 g(n)>\frac{35}{32} n-\frac{3}{2}$, for $n \geq 4$.

Proof 4 For $n \geq 48$, let $n=16 h+c$ with $0 \leq c<16$. By Theorem 5 we get $g(n) \geq g(16 h)+c \geq(35 / 32) 16 h+(35 / 32) c-(35 / 32-1) c=(35 / 32) n-$ $(3 / 32) c>(35 / 32) n-3 / 2$. For $9 \leq n \leq 47$, note that the bound on $g_{3}(n)$ in Theorem 6 is better than the bound on $g(n)$ in Theorem 8 for these values of $n$, i.e., $n-4+\sqrt{2(n-3)}>(35 / 32) n-3 / 2$ for $9 \leq n \leq 47$. By definition $g(n)$ and $g_{3}(n)$ are the maximum values of $G(V)$ over two classes of sets, one containing the other, hence $g(n)$ and $g_{3}(n)$ satisfy $g(n) \geq g_{3}(n)$ for all $n$. Therefore in this case $g(n)>(35 / 32) n-3 / 2$ by virtue of Theorem 6 . The case $4 \leq n \leq 8$ can be verified using Theorem 4 for $k=1,2$ and the monotonicity of $g$.

\section{Conjectures}

We conjecture that our bound for $g_{3}$ (Theorem 6) is tight up to a constant. Also we note that our basic construction seems to admit a direct generalization to higher dimensions and thus it is likely that the results and proofs concerning this construction (i.e., up to Theorem 6) might also have a generalization.

Acknowledgments We are grateful to the anonymous referees for their suggestions and corrections.

\section{References}

1. Aichholzer, O., Huemer, C., Kappes, S., Speckmann, B., Tóth, C.D.: Decompositions, partitions, and coverings with convex polygons and pseudo-triangles. Graphs Combin. 23(5), 481-507 (2007)

2. Aichholzer, O., Krasser, H.: The point set order type data base: a collection of applications and results. In: Proc. 13th Ann. Canadian Conference on Computational Geometry, pp. 17-20. Waterloo, Canada (2001)

3. Brass, P., Moser, W., Pach, J.: Research Problems in Discrete Geometry. Springer, New York (2005)

4. García-López, J., Nicolás, C.M. : Planar point sets with large minimum convex partitions. In: Abstracts 22nd European Workshop on Comput. Geom., pp. 51-54. Delphi, Greece (2006)

5. Hosono, K.: On convex decompositions of a planar point set. Discret. Math. 309(6), 1714-1717 (2009)

6. Lee, C.W.: Subdivisions and triangulations of polytopes. In: Handbook of Discrete and Computational Geometry. CRC Press Ser. Discret. Math. Appl., pp. 271-290. CRC Press, Boca Raton (1997)

7. Sakai, T., Urrutia, J.: Convex decompositions of point sets in the plane. In: Abstracts 7th Japan Conference on Computational Geometry and Graphs. Kanazawa, Japan (2009) 